\section{Current role of computed tomography-guided transthoracic needle biopsy of metastatic lung lesions}

\author{
Anna Galluzzo ${ }^{*}$, , Claudio Genova ${ }^{2}$, Salvatore Dioguardi ${ }^{3}$, Massimo Midiri² \\ \& Massimo Cajozzo 3
}

\begin{abstract}
Aim: As part of the Catania symposium on lung metastasectomy we reviewed our practice of computed tomography (CT)-guided percutaneous transthoracic needle biopsy of pulmonary metastatic lesions with particular emphasis on diagnostic accuracy and nature of complications lesions. Materials \& methods: 25 patients with metastatic lesions of the lung have been evaluated between May 2010 and February 2014. Inclusion criteria consisted of patients with histologically confirmed, metastatic disease of the lung, those receiving a CT-guided needle biopsy, were at least 18 years of age; and with adequate hepatic, renal and hematological function. We recorded also the size of the sampled lesions, their distance from the pleura, the complications encountered (pneumothorax and thoracostomy tube placement), the cytological diagnosis and the outcome in all the cases. Results: CT-guided percutaneous transthoracic needle biopsy were performed on 23 of 25 patients with suspected lung metastases. 17 males and six females with a mean age of 71.4 years. The mean size of lesions was $4.2 \mathrm{~cm}$ (range: 1 to $17 \mathrm{~cm}$ ). For CT-guided needle biopsy, an 18 gauge semi-automatic needle biopsy device was used. Of 23 biopsies, 20 (87\%) yielded a correct diagnosis with specific histological typing for metastasis. Pneumothorax was the most common complication occurring in four cases (5.7\%). Conclusion: CT-guided percutaneous transthoracic needle biopsy is a firm, useful and safe technique for the diagnosis of suspected pulmonary metastases as it avoids open biopsy in most cases.
\end{abstract}

It is known that the lungs are a common site for metastases from other primary cancers, such as breast, colon and sarcomas. Although in the patients with high-risk of lung metastasis the increasing utility of computed tomography (CT) low-dose screening has grown dramatically the sensitivity of identifying small lung nodules [1], CT-guided needle biopsy (CTgNB) of lung metastases (LM) with automatic or semi-automatic biopsy gun have proven to bring in diagnostic label with few complications and is widely accepted as accurate and safe procedure for characterizing pulmonary nodules. [2]

Percutaneous transthoracic needle biopsy guided by CT is considered as a relatively well and safe method for diagnosing LM [3]. The rates of diagnostic accuracy have been documented between 72 and $100 \%$ for pulmonary metastatic lesions. However, the diagnostic accuracy decrease for smaller lesions between 10 and $20 \mathrm{~mm}$; several studies have reported the diagnostic accuracy range of 52 and $96.5 \%[4,5]$.

The purpose of our study was to review our experience with CT-guided biopsy of suspected LM with a view to providing a critical assessment of the efficacy and safety of the procedure.

IBFM-CNR at Department of Radiology (Department of Biotechnology, Biopathology and Forensic Medicine), University of Palermo, via del Vespro 127, 90129 Palermo, Italy

${ }^{2}$ Department of Radiology, University of Palermo, Italy

${ }^{3}$ Department of Thoracic Surgery, University of Palermo, Italy

*Author for correspondence: Tel.: +39 3474224353; Fax: +39 0916552332; anna.galluzzo@gmail.com

\section{KEYWORDS}

- CT-guided biopsy • lung metastasis $\bullet$ transthoracic needle biopsy 


\section{Materials \& methods}

Twenty-five patients with metastatic lesions of the lung larger than $3 \mathrm{~cm}$ have been evaluated between May 2010 and February 2014.

Inclusion criteria were patients with histologically confirmed cancer and suspected metastatic disease of the lung; 18 years of age and adequate hepatic, renal and hematological function.

Exclusion criteria were inadequacy of the sample taking, substantial medical comorbidities. Patients receiving warfarin or low-molecular-weight heparin were eligible, and were asked to stop warfarin therapy 1 week before the procedure and monitoring of prothrombin time and bleeding risks. Platelet counts of $\geq 1.0 \times 10^{9} / \mathrm{ml}$ and $\geq 50 \times 10^{6} / \mathrm{ml}$, respectively, were required for biopsy.

Collected data included the patient's age and gender, primary cancer, as well pathology record to the $\mathrm{C} \operatorname{TgNB}$, and the last available follow-up to evaluate the contingent periprocedural complications. We recorded also the size of the sampled lesions, their distance from the pleura, the complications encountered (pneumothorax and thoracostomy tube placement), the cytological diagnosis and the outcome in all the cases.

This single institutional retrospective study was approved by our institutional review board (IRB). All patients were required to provide a written informed consent before underwent a CTgNB in accordance with institutional guidelines.

\section{CT-guided procedure}

The CT images at the time of biopsy were retrospectively reviewed by two radiologist (AG and CG). A coaxial technique was performed in all the cases, and a single needle (18-gauge Menghini semi-automic cutting with coaxial technique) was used in all cases (100\%), whereas different needles in combination were used when multiple samples had to be obtained.

The appropriate length of needles was chosen based on the depth of the lesion from the skin as measured by using the CT images. Needle biopsy was performed under guidance of a Multidetector CT on a 16 (Speedlight, GE Healthcare, WI, USA) slice scanner. Low-dose (120 kVp, $30 \mathrm{~mA}$ ) CT images were obtained through the area of interest using a section thickness of $2.5-5 \mathrm{~mm}$. Localization was determined by the CT gantry laser lights and grid on the skin. The skin entry site was sterilized, draped and local anesthesia was used.

After needle insertion, CT images were obtained at $1.5-\mathrm{mm}$ section thicknesses to check the position of the needle tip. After confirmation of adequate position of the needle tip, core biopsy were obtained and the CTgNB specimens transferred onto microscopy slides were read by a cytopathologist. After removal of the needle, a minimum dose CT of the thorax $(120 \mathrm{kV}$, $10 \mathrm{~mA}, 8$-mm section thickness) was performed to assess for pneumothorax. Patients were subsequently monitored by nurses in the Thoracic Surgery Department Day Unit.

\section{Results}

23 of 25 patients with suspected LM have been evaluated, between them two patients did not have biopsy because procedural difficulties and therefore 23 patients have been included in the study. There were 17 males and six females, with a mean age of 71.4 years (range: 54-87). In one patient, the biopsy was repeated because of inadequacy of the first sample. Six patients underwent open surgical wedge resection of the metastases, and 17 nonsurgical patients who had been followed up for at least 3 months.

Radiologically, the lesions appeared as a solid mass in 22 cases $(95.6 \%)$ or a cystic mass in one $(4.4 \%)$. The mean diameter of the lesion was $4.2 \mathrm{~cm}$ ranged from 1 to $17 \mathrm{~cm}$ (mean $4.2 \mathrm{~cm} ; \mathrm{SD} \pm 1.99$ ). 13 lesions were located in the superior lobes (56.5\%); nine lesions [39.1\%] in the left superior lobe and four $(17.4 \%)$ in the right superior lobe. Five lesions in the inferior lobes $(21.7 \%)$, of those three were $(13 \%)$ in the left lower lobe and two (8.6\%) in the right lower lobe.

To calculate the diagnostic accuracy, all patients were included. In 20 (87\%) out 23 biopsies, a correct diagnosis with specific histological typing for metastasis was made. The overall sensitivity was $91.8 \%$.

In the six operated patients diagnosis was confirmed and one extra nodule was found. Histology showed metastasis of colon cancer (CDX2 and CK20+) in nine patients, of carcinoma of the endometrium in five, sarcomas in five (one sarcomatoid carcinoma G-3, one leiomyosarcoma, two condrosarcomas) and undifferentiated carcinoma in three.

Of the three negative patients, one had the final diagnosis of inflammatory bronchiolitis disease and a second patient had a diagnosis of Langherans Cell Histiocytosis. Pneumothorax was the most common complication and occurred in four cases (17.4\%), no hemorrhage was recorded. 


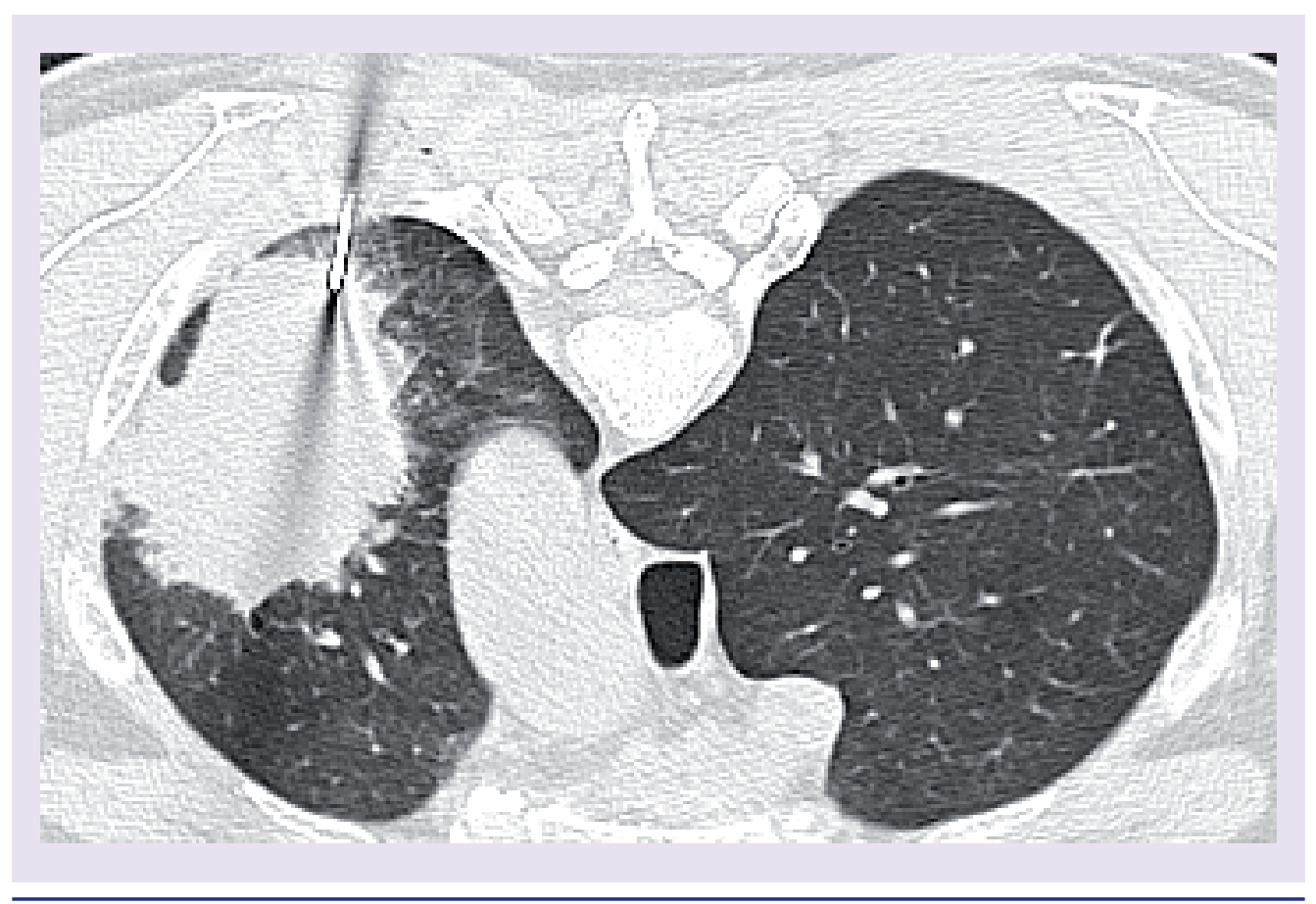

Figure 1. Unenhanced computed tomography axial scan in a 65-year-old male. A large mass is adjacent to the left pleura. The tip of the needle is well visible inside the mass. The histological examination proved the lesion to be lung metastases of colon adenocarcinoma.

\section{Discussion}

Metastatic disease is the main cause of death owing to cancer, and the lung is a common site of distant metastases [6]. When a new nodule appears in the lung in patients with operated primary tumor, cancer progression is generally thought and definite diagnosis is mandatory to determine whether the lesion is malignant or benign. Even when a lesion appears to be either malignant or benign on radiography, histological specimens should confirm the final diagnosis [7]. Preoperative diagnosis is in fact standard practice but is also a mandatory to include patients in prospective randomized trial that have the goal to demonstrate the effectiveness of lung biopsy for metastasis [8-11].

CT-guided biopsy represents therefore an important step to establish the diagnosis, and MDCT is superior to conventional radiography for detecting small pulmonary metastasis.

The CTgNB system provides microhistological samples to confirm the diagnosis of LM, which is crucial for planning an appropriate treatment. Because most of our cases have a large metastases (mean size is above $4 \mathrm{~cm}$ ) the diagnostic accuracy has been high [12]. Moreover, the preoperative diagnosis of large mass (Figure 1) suspected for LM is of paramount importance as extended operations for LM have been reported with poor outcome [13]. We performed open surgical resection as in absence of proved advantage of one open versus VATS metastasectomy [4,14], we prefer to palpate all the lung to search for extra nodules.

In our small experience we reveal that the most common histology of secondary tumors were colon adenocarcinoma and pneumothorax was the most common complication occurring in 17\% (95\% CI: 7-37\%) of our patients which is within the $5-65 \%$ reported incidence in the literature.

A recent study on 15.865 adults who had transthoracic needle biopsy of a pulmonary nodule showed that the risk for any pneumothorax was $15.0 \%$ (CI: $14.0-16.0 \%$ ), and 6.6\% (CI: $6.0-7.2 \%)$ of all biopsies resulted in pneumothorax requiring a chest tube [15]. On the contrary, we did not insert chest drains but again our experience is small. The fact that the rate of pneumothorax in our experience is so low is probably due to the fact that the biopsied lesions are large and most adherent to the chest wall.

Only in two patients we have performed open biopsy to reach the diagnosis.

\section{Conclusion}

CT-guided percutaneous transthoracic needle biopsy is a firm, useful and safe technique for the 
diagnosis of suspected pulmonary metastases as it avoids open biopsy in most cases.

Financial \& competing interests disclosure

The authors have no relevant affiliations or financial involve-

ment with any organization or entity with a financial interest in or financial conflict with the subject matter or materials discussed in the manuscript. This includes employment, consultancies, honoraria, stock ownership or options, expert testimony, grants or patents received or pending, or royalties.

No writing assistance was utilized in the production of this manuscript.

\section{References}

1 Henschke CI, McCauley DI, Yankelevitz DF et al. Early Lung Cancer Action Project: overall design and findings from baseline screening. Lancet 354, 99-105 (1999).

2 Todd TRJ, Weisbrod GL, Tao LC et al. Aspiration needle biopsy of thoracic lesions. Ann. Thorac. Surg. 32, 154-161 (1981).

3 Li H, Boiselle PM, Shepard JO, Trotman-Dickenson B, McLoud TC. Diagnostic accuracy and safety of CT-guided percutaneous needle aspiration biopsy of the lung: comparison of small and large pulmonary nodules. Am. J. Roentgenol. 167, 105-109 (1996).

4 Ohno H, Hatabu D, Takenaka et al. CT-guided transthoracic needle aspiration biopsy of small $(\leq 20 \mathrm{~mm})$ solitary pulmonary nodules. Am. J. Roentgenol. 180, 1665-1669 (2003).

5 Tsukada H, Satou T, Iwashima A, Souma T. Diagnostic accuracy of CT-guided automated needle biopsy of lung nodules. $\mathrm{Am}$. $\mathrm{J}$. Roentgenol. 175(1), 239-243 (2000).
Coghlin C, Murray. GI. The role of gene regulatory networks in promoting cancer progression and metastasis. Future Oncol. 10(5), 735-748 (2014).

7 Bladt O, De Wever W. Additional value of ct-fluoroscopic biopsy of pulmonary lesions: a retrospective study of 69 patients. JBR-BTR 89, 298-302 (2006).

8 Treasure T, Fiorentino F, Scarci M, Møller H, Utley M. Pulmonary metastasectomy for sarcoma: a systematic review of reported outcomes in the context of Thames Cancer Registry data. BMJ Open 2, e001736 (2012)

9 Treasure T, Milosevic M, Fiorentino F, Macbeth F. Pulmonary metastasectomy: what is the practice and where is the evidence for effectiveness? Thorax doi:10.1136/ thoraxjnl-2013-204528 (2014).

10 Fiorentino F, Treasure T. Pulmonary metastasectomy for colorectal cancer: making the case for a randomized controlled trial in the zone of uncertainty. J. Thorac. Cardiovasc. Surg. 146(4), 748-752 (2013).
11 Treasure T, Miloševic M, Migliore M, Lees B. Pulmonary Metastasectomy in Colorectal Cancer (PulMiCC International). Colorectal Cancer 2(6), 505-513 (2013).

12 Kothary N, Lock L, Sze DY, Hofmann LV. Computed tomography-guided percutaneous needle biopsy of pulmonary nodules: impact of nodule size on diagnostic accuracy. Clin. Lung Cancer 10 (5), 360-363 (2009).

13 Migliore M, Jakovic R, Hensens A, Klepetko W. Extending surgery for pulmonary metastasectomy: what are the limits? J. Thorac. Oncol. 5(6), S155-S160 (2010).

14 Migliore M, Criscione A, Calvo D et al. Wider implications of video-assisted thoracic surgery versus open approach for lung metastasectomy. Future Oncol. 11(Suppl. 2), 25-29 (2014)

15 Wiener RS, Schwartz LM, Woloshin S, Welch HG. Population-based risk for complications after transthoracic needle lung biopsy of a pulmonary nodule: an analysis of discharge records. Ann. Intern. Med. 155(3), 137-144 (2011). 